\title{
Past, present, and future of donation after circulatory death in Italy
}

\author{
Luciano De Carlis ${ }^{1,2} \cdot$ Riccardo De Carlis $^{1,3}$ (D) Paolo Muiesan ${ }^{4,5}$ (1)
}

Received: 20 February 2019 / Accepted: 8 March 2019 / Published online: 12 March 2019

(C) Italian Society of Surgery (SIC) 2019

Keywords Solid organ transplantation · Organ recovery $\cdot$ Normothermic regional perfusion $\cdot$ Machine perfusion . No-touch period

Different strategies have been advocated to overcome the shortage of organs for transplantation. In addition to accepting extended criteria organs from donation after brain death (DBD) and promoting living donation, donation after circulatory death (DCD) is an option worth considering. This is confirmed by the policy of some European countries, such as the United Kingdom, where, in 2018, DCD reached a peak of $40 \%$ of all deceased donation [1, 2]. In Italy, the declaration of death based on circulatory criteria requires a no-touch period of at least $20 \mathrm{~min}$, much longer compared to the $5 \mathrm{~min}$ accepted in other countries. Concerns about warm ischemia arising from this legal constraint have long discouraged the development of a DCD transplant program in Italy. A handful of DCD kidneys were recovered with the superrapid technique and transplanted, but this practice was soon abandoned after the enactment of brain death legislation.

Nevertheless, in 2007, a pilot project on kidney transplants from uncontrolled DCD donors was initiated in Pavia using normothermic regional perfusion (NRP) between death declaration and organ recovery [3]. In 2014, Valenza et al. started a protocol to procure lungs from uncontrolled DCD that included donor maintenance on mechanical

Riccardo De Carlis

riccardo.decarlis@ospedaleniguarda.it

1 Department of General Surgery and Transplantation, ASST Grande Ospedale Metropolitano Niguarda, Piazza Ospedale Maggiore 3, 20162 Milan, Italy

2 School of Medicine and Surgery, University of Milano-Bicocca, Milan, Italy

3 Department of Surgical Sciences, University of Pavia, Pavia, Italy

4 Liver Unit, University Hospitals Birmingham NHS Foundation Trust, Birmingham, UK

5 Hepatobiliary Unit, Careggi University Hospital, University of Florence, Florence, Italy ventilation only and subsequent evaluation of the recovered lungs with ex vivo perfusion. Over 10 cases, only one underwent the full process and was transplanted, showing good graft function after a six-month follow-up [4]. One year later, the team of Niguarda Hospital performed the first successful DCD liver transplant in Italy from a donor maintained on NRP [5]. The same group implemented the initial protocol with the use of hypothermic oxygenated machine perfusion (HOPE) after NRP [6, 7]. Novel protocols including normothermic machine perfusion or the combined recovery of thoracic and abdominal organs during NRP are currently under development in Italy.

Abdominal NRP relies on an extracorporeal circuit made up of a centrifugal pump and a membrane oxygenator. The donor's femoral vessels are cannulated, and a Fogarty balloon is inflated in the supraceliac aorta to exclude the chest and avoid reperfusion of the brain. In Italy, percutaneous insertion of introducer sheaths is allowed pre-mortem to facilitate vessel cannulation, which is performed after death has been declared. In this way, NRP acts as a perfusion bridge between cardiac arrest and organ recovery, allowing the repletion of cellular energy stores after warm ischemia and an initial assessment of liver function [8, 9]. Moreover, different types of organ machine perfusion (MP), ranging from hypothermia to normothermia, have been recently introduced in clinical practice. The combined use of in situ and ex situ perfusion offers potential advantages of particular importance for DCD transplantation: organ reconditioning, viability testing and improved preservation times which favour transplant logistics [10].

The feasibility of DCD liver transplantation despite a no-touch period of 20 min has drawn renewed interest in the DCD practice in Italy. But was DCD just a one-day wonder or does it have a future in this country? To answer the question, two elements must be considered: the outcomes of transplants from DCD and the current trend in 
DCD donations. The inferior graft survival compared with DBD donors and the increased incidence of ischemic cholangiopathy (IC) are traditionally the two main deterrents to DCD liver transplantation and the 20-min no-touch period is expected to increase these complications [11]. Nevertheless, the latest analysis of the series of Niguarda Hospital has shown no difference in 1-year patient survival compared to DBD grafts (95\% vs. $94 \%, p=0.94)$ and a slightly inferior 1-year graft survival in the DCD group $(85 \%$ vs. $91 \%$, $p=0.20$ ). Interestingly, IC did not adversely impact graft survival, as none of the recipients underwent retransplantation due to biliary complications [12]. Ravaioli et al. have recently published a series of 10 kidneys from controlled DCD donors using NRP and HOPE. The authors reported a delayed graft function rate of $30 \%$ but no cases of primary non-function [13].

The number of utilized DCD donors in Italy has progressively risen from 14 in 2016 to 32 in 2017, and 47 in 2018 [14]. There are three possible scenarios: uncontrolled DCD, which occurs in case of out-of-hospital cardiac arrest; controlled DCD, which occurs after a planned withdrawal of life-supporting therapies; and DCD during extracorporeal life support (ECLS), which occurs when ECLS, previously started with resuscitative intent, is then stopped due to futility or impossibility to maintain circulation and converted to NRP. The majority of DCD donors in Italy are managed in a small number of donor hospitals with expertise in ECLS and NRP [15]. These are geographically remote to liver transplant centers willing to accept DCD livers. This is an important logistical problem, particularly in case of uncontrolled donors. A shared donor management policy between the DCD donor hospitals and the transplant centers is of paramount importance for the project and should be the goal of the future policy on DCD in Italy.

Therefore, DCD in Italy is inseparable from NRP, and most transplant protocols include the use of MP for graft reconditioning and evaluation. There is no doubt that NRP and MP bring into the picture considerable costs in terms of equipment and personnel. This raises some questions about the cost-effectiveness of DCD. Some years ago, Cavallo et al. have shown that increasing DCD kidney transplant can result in a cost-effective policy [16]. This economic analysis was performed when kidney transplants were the only transplants from DCD performed in Italy. Every DCD donor should be now considered also for liver, lung, and more rarely pancreatic islet cell transplantation. It seems, thus, reasonable that the benefit could be even greater.

In conclusion, although still in its infancy and despite the difficulties related to the no-touch period of $20 \mathrm{~min}$, DCD in Italy has provided good results, produced innovative protocols based on the most modern combination of preservation techniques and has the potential to further increase the number of organs available for transplantation.

\section{Compliance with ethical standards}

Conflict of interest The authors have no conflict of interest or funding to declare.

Research involving human participants and/or animals As a commentary article, no ethical approval is required.

Informed consent As a commentary article, no informed consent was obtained.

\section{References}

1. NHS Blood and Transplant. Organ Donation and Transplantation Activity Report 2017/18. https://nhsbtdbe.blob.core.windows.net/ umbraco-assets-corp/12300/transplant-activity-report-2017-2018. pdf. Accessed 18 Feb 2019

2. Schlegel A, Kalisvaart M, Scalera I, Laing RW, Mergental H, Mirza DF, Perera T, Isaac J, Dutkowski P, Muiesan P (2018) The UK DCD Risk Score: a new proposal to define futility in donation-after-circulatory-death liver transplantation. J Hepatol 68(3):456-464. https://doi.org/10.1016/j.jhep.2017.10.034

3. Geraci PM, Sepe V (2011) Non-heart-beating organ donation in Italy. Minerva Anestesiol 77(6):613-623

4. Valenza F, Citerio G, Palleschi A et al (2016) Successful transplantation of lungs from an uncontrolled donor after circulatory death preserved in situ by alveolar recruitment maneuvers and assessed by ex vivo lung perfusion. Am J Transplant 16(4):13121318. https://doi.org/10.1111/ajt.13612

5. De Carlis L, Lauterio A, De Carlis R, Ferla F, Di Sandro S (2016) Donation after cardiac death liver transplantation after more than 20 minutes of circulatory arrest and normothermic regional perfusion. Transplantation 100(4):e21-e22. https://doi.org/10.1097/ TP.0000000000001136

6. De Carlis L, De Carlis R, Lauterio A, Di Sandro S, Ferla F, Zanierato $M$ (2016) Sequential use of normothermic regional perfusion and hypothermic machine perfusion in donation after cardiac death liver transplantation with extended warm ischemia time. Transplantation 100(10):e101-e102. https://doi.org/10.1097/ TP.0000000000001419

7. De Carlis R, Di Sandro S, Lauterio A, Ferla F, Dell'Acqua A, Zanierato M, De Carlis L (2017) Successful donation after cardiac death liver transplants with prolonged warm ischemia time using normothermic regional perfusion. Liver Transplant 23(2):166173. https://doi.org/10.1002/lt.24666

8. Lazzeri C, Bonizzoli M, Valente S, Cianchi G, Migliaccio ML, Gensini GF, Peris A (2014) The role of extracorporeal membrane oxygenation in donation after circulatory death. Minerva Anestesiol 80(11):1217-1227

9. Peris A, Lazzeri C, Bonizzoli M et al (2018) A metabolic approach during normothermic regional perfusion in uncontrolled donors after circulatory death-a pilot study. Clin Transplant 32(10):e13387. https://doi.org/10.1111/ctr.13387

10. De Carlis R, Lauterio A, Ferla F, Di Sandro S, Sguinzi R, De Carlis L (2017) Hypothermic machine perfusion of liver grafts can safely extend cold ischemia for up to 20 hours in cases of necessity. Transplantation 101(7):e223-e224. https://doi.org/10.1097/ TP.0000000000001753

11. O'Neill S, Roebuck A, Khoo E, Wigmore SJ, Harrison EM (2014) A meta-analysis and meta-regression of outcomes including biliary complications in donation after cardiac death liver 
transplantation. Transpl Int 27(11):1159-1174. https://doi. org/10.1111/tri.12403

12. De Carlis R, Di Sandro S, Lauterio A, Botta F, Ferla F, Andorno E, Bagnardi V, De Carlis L (2018) Liver grafts from donors after circulatory death on regional perfusion with extended warm ischemia compared with donors after brain death. Liver Transpl 24(11):1523-1535. https://doi.org/10.1002/lt.25312

13. Ravaioli M, De Pace V, Comai G et al (2018) Preliminary experience of sequential use of normothermic and hypothermic oxygenated perfusion for donation after circulatory death kidney with warm ischemia time over the conventional criteria-a retrospective and observational study. Transpl Int 31(11):1233-1244. https ://doi.org/10.1111/tri.13311

14. Italian National Transplant Center. Donation and transplant activity of organs, tissues and hematopoietic stem cells-Report 2018. http://www.trapianti.salute.gov.it/imgs/C_17_cntPubblicazion i_266_allegato.pdf. Accessed 2 Mar 2019
15. Peris A, Lazzeri C, Cianchi G et al (2018) Implementing a donation after circulatory death program in a setting of donation after brain death activity. Minerva Anestesiol 84(12):1387-1392. https ://doi.org/10.23736/S0375-9393.18.12635-6

16. Cavallo MC, Sepe V, Conte F, Abelli M, Ticozzelli E, Bottazzi A, Geraci PM (2014) Cost-effectiveness of kidney transplantation from DCD in Italy. Transplant Proc 46(10):3289-3296. https://doi. org/10.1016/j.transproceed.2014.09.146

Publisher's Note Springer Nature remains neutral with regard to jurisdictional claims in published maps and institutional affiliations. 